\title{
ON THE DERIVATIVES OF ZETA-FUNCTIONS OF CERTAIN CUSP FORMS, II
}

\author{
A. LAURINČIKAS \\ Department of Mathematics and Informatics, Vilnius University, Naugarduko 24, 3225 Vilnius, Lithuania \\ Department of Physics and Mathematics, Šiauliai University, Višinskio 19, 5440 Šiauliai, Lithuania \\ e-mail: antanas.laurincikas@maf.vu.lt
}

(Received 26 January, 2005; accepted 27 May, 2005)

\begin{abstract}
The discrete universality of the derivative and logarithmic derivative of zeta-functions of normalized eigenforms is obtained. This is used to estimate the number of zeros of the derivatives in the critical strip. For the proof the method of functional limit theorems in the sense of weak convergence of probability measures is applied.
\end{abstract}

2000 Mathematics Subject Classification. 11 M41.

1. Introduction. Let $F(z)$ be a normalized eigenform of weight $\kappa$ for all Hecke operators. Then $F(z)$ has the Fourier series expansion

$$
F(z)=\sum_{m=1}^{\infty} c(m) e^{2 \pi i m z}
$$

with multiplicative coefficients $c(m)$. Denote by $s=\sigma+i t$ a complex variable, and attach to the form $F(z)$ the zeta-function

$$
\varphi(s ; F)=\sum_{m=1}^{\infty} \frac{c(m)}{m^{s}} .
$$

In view of multiplicativity of the coefficients $c(m)$, the function $\varphi(s ; F)$ has the Euler product expansion over primes

$$
\varphi(s ; F)=\prod_{p}\left(1-\frac{\alpha(p)}{p^{s}}\right)^{-1}\left(1-\frac{\beta(p)}{p^{s}}\right)^{-1},
$$

where $\alpha(p)+\beta(p)=c(p)$. By Deligne's estimates [2] both the Dirichlet series and product converge absolutely in the half-plane $\sigma>\frac{\kappa+1}{2}$ and define there a holomorphic function with no zeros. Moreover, the function $\varphi(s ; F)$ is analytically continuable to an entire function and satisfies the functional equation of the Riemann type

$$
(2 \pi)^{-s} \Gamma(s) \varphi(s ; F)=(-1)^{\kappa / 2}(2 \pi)^{s-\kappa} \Gamma(\kappa-s) \varphi(\kappa-s ; F) .
$$

S. M. Voronin [14] was the first to prove (continuous) universality for the Riemann zeta-function, and discrete universality was invented by A. Reich [13] for Dedekind 
zeta-functions. In [5] we began to study value-distribution of the derivative as well as of the logarithmic derivative of the function $\varphi(s ; F)$. We proved the universality in the Voronin sense [14] for the functions $\varphi^{\prime}(s ; F)$ and $\frac{\varphi^{\prime}}{\varphi}(s ; F)$, and obtained that $\varphi^{\prime}(s ; F)$ has infinitely many zeros in the critical strip $\{s \in \mathbb{C}: \kappa / 2<\sigma<(\kappa+1) / 2\}$. To state more precisely the mentioned results we need some notation. Denote by meas $\{A\}$ the Lebesgue measure of a measurable set $A \subset \mathbb{R}$, and let, for $T>0$,

$$
v_{T}(\ldots)=\frac{1}{T} \operatorname{meas}\{\tau \in[0, T]: \ldots\},
$$

where in place of dots a condition satisfied by $\tau$ is to be written. Then in [5] the following statements have been obtained. Let $D=\{s \in \mathbb{C}: \kappa / 2<\sigma<(\kappa+1) / 2\}$, and let $\psi(s ; F)$ denote $\varphi^{\prime}(s ; F)$ or $\frac{\varphi^{\prime}}{\varphi}(s ; F)$.

THEOREM A. Let $K$ be a compact subset of the strip $D$ with connected complement, and let $f(s)$ be a continuous on K function which is analytic in the interior of $K$. Then, for every $\varepsilon>0$,

$$
\liminf _{T \rightarrow \infty} \nu_{T}\left(\sup _{s \in K}|\psi(s+i \tau ; F)-f(s)|<\varepsilon\right)>0 .
$$

THEOREM B. For every $\sigma_{1}, \sigma_{2}, \kappa / 2<\sigma_{1}<\sigma_{2}<(\kappa+1) / 2$, there exists a constant $c=c\left(\sigma_{1}, \sigma_{2}\right)>0$ such that, for sufficiently large $T$, the function $\varphi^{\prime}(s ; F)$ has more than $c T$ zeros in the rectangle

$$
\left\{s \in \mathbb{C}: \sigma_{1}<\sigma<\sigma_{2}, 0<t<T\right\} .
$$

In Theorem A the translation $\tau$ varies continuously in the interval $[0, T]$; therefore Theorem A can be called the continuous universality theorem. The aim of this note is to obtain discrete versions of Theorems $\mathrm{A}$ and $\mathrm{B}$, when the translation $\tau$ takes values from a certain arithmetical progression with the first term zero and difference $h>0$. Let, for $N \in \mathbb{N}$,

$$
\mu_{N}(\ldots)=\frac{1}{N+1} \#\{0 \leq m \leq N: \ldots\}
$$

where in place of dots a condition satisfied by $m$ is to be written.

THEOREM 1. Let $K$ be a compact subset of the strip $D$ with connected complement, and let $f(s)$ be a function continuous on $K$ which is analytic in the interior of $K$. Then, for every $\varepsilon>0$ and arbitrary positive real number $h$,

$$
\liminf _{N \rightarrow \infty} \mu_{N}\left(\sup _{s \in K}|\psi(s+i m h ; F)-f(s)|<\varepsilon\right)>0 .
$$

The function $\psi(s, F)$ does not have an Euler product. Therefore, the function $f(s)$ in Theorem 1 is not necessarily non-vanishing.

THEOREM 2. For arbitrary $\sigma_{1}, \sigma_{2}, \kappa / 2<\sigma_{1}<\sigma_{2}<(\kappa+1) / 2$, there exists a constant $c=c\left(\sigma_{1}, \sigma_{2}\right)>0$ such that, for sufficiently large $N$, the function $\varphi^{\prime}(s+i m h ; F)$ has a zero on the disc $|s-\widehat{\sigma}| \leq\left(\sigma_{2}-\sigma_{1}\right) / 2, \widehat{\sigma}=\frac{\sigma_{1}+\sigma_{2}}{2}$, for more than $c N$ numbers $m, 0 \leq m \leq N$.

We note that in this paper there is no restriction on $h$ as in former results [10]. 
We consider two cases. In the first case we assume the number $\exp \left\{\frac{2 \pi k}{h}\right\}$ to be irrational for all $k \in \mathbb{Z} \backslash\{0\}$, while in the second case this number is supposed to be rational for some $k \neq 0$. In the first case, Theorem 1 with $\psi(s ; F)=\varphi^{\prime}(s ; F)$ and Theorem 2 were proved in [10] if $F$ is a newform of weight $\kappa$ and level $q$. So, it remains to obtain Theorem 1 with $\psi(s ; F)=\frac{\varphi^{\prime}}{\varphi}(s, F)$ in the first case, and Theorems 1 and 2 in the second case.

The proof of Theorem 1 uses Bagchi's method based on limit theorems in the sense of weak convergence of probability measures in the spaces of analytic and meromorphic functions. An application of Theorem 1 together with Rouchés theorem yields Theorem 2 .

2. A discrete limit theorem for $\frac{\varphi^{\prime}}{\varphi}(s, \boldsymbol{F})$. Case 1. Let $\gamma=\{s \in \mathbb{C}:|s|=1\}$ be the unit circle on the complex plane. As usual, we define a probability space $\left(\Omega, \mathcal{B}(\Omega), m_{H}\right)$, where

$$
\Omega=\prod_{p} \gamma_{p}
$$

with $\gamma_{p}=\gamma$ for each prime $p, \mathcal{B}(S)$ denotes the class of Borel sets of the space $S$, and $m_{H}$ is the probability Haar measure on $(\Omega, \mathcal{B}(\Omega))$. The measure $m_{H}$ can be defined because $\Omega$ with the product topology and pointwise multiplication is a compact topological Abelian group. Denote by $\omega(p)$ the projection of $\omega \in \Omega$ to the coordinate space $\gamma_{p}$.

Let $\mathbb{C}_{\infty}=\mathbb{C} \cup\{\infty\}$ be the Riemann sphere with spheric metric $d$ defined by the formulae

$$
d\left(s_{1}, s_{2}\right)=\frac{2\left|s_{1}-s_{2}\right|}{\sqrt{1+\left|s_{1}\right|^{2}} \sqrt{1+\left|s_{2}\right|^{2}}}, \quad d(s, \infty)=\frac{2}{\sqrt{1+|s|^{2}}}, \quad d(\infty, \infty)=0
$$

$s, s_{1}, s_{2} \in \mathbb{C}$. By $M(G)$ denote the space of meromorphic on $G$ functions $g: G \rightarrow$ $\left(\mathbb{C}_{\infty}, d\right)$ equipped with the topology of uniform convergence on compacta. In this topology, a sequence $g_{n}(s) \in M(G)$ converges to $g(s) \in M(G)$ if

$$
d\left(g_{n}(s), g(s)\right) \longrightarrow 0, \quad n \rightarrow \infty
$$

uniformly on compact subsets of $G$. The space $H(G)$ of analytic on $G$ functions is a subspace of $M(G)$.

For $V>0$, let $D_{V}=\left\{s \in \mathbb{C}: \frac{\kappa}{2}<\sigma<\frac{\kappa+1}{2},|t|<V\right\}$. On the probability space $\left(\Omega, \mathcal{B}(\Omega), m_{H}\right)$ define an $H\left(D_{V}\right)$-valued random element $\frac{\varphi^{\prime}}{\varphi}(s, \omega ; F)$ by

$$
\begin{aligned}
\frac{\varphi^{\prime}}{\varphi}(s, \omega ; F)= & -\sum_{p} \frac{\alpha(p) \omega(p) \log p}{p^{s}}\left(1-\frac{\alpha(p) \omega(p)}{p^{s}}\right)^{-1} \\
& -\sum_{p} \frac{\beta(p) \omega(p) \log p}{p^{s}}\left(1-\frac{\beta(p) \omega(p)}{p^{s}}\right)^{-1}
\end{aligned}
$$


THEOREM 3. Let $\exp \left\{\frac{2 \pi k}{h}\right\}$ be irrational for all $k \in \mathbb{Z} \backslash\{0\}$. Then the probability measure

$$
\mu_{N}\left(\frac{\varphi^{\prime}}{\varphi}(s+i m h ; F) \in A\right), \quad A \in \mathcal{B}\left(M\left(D_{V}\right)\right),
$$

converges weakly to the distribution of the random element $\frac{\varphi^{\prime}}{\varphi}(s, \omega ; F)$ as $N \rightarrow \infty$.

For the proof of Theorem 3 we will use the following statement obtained in [12, Lemma 1], in the case of newforms.

LeMma 4. As $N \rightarrow \infty$, the probability measure

$$
\mu_{N}(\varphi(s+i m h ; F) \in A), \quad A \in \mathcal{B}\left(H\left(D_{V}\right)\right),
$$

converges weakly to the distribution of the $H\left(D_{V}\right)$-valued random element

$$
\varphi(s, \omega ; F)=\prod_{p}\left(1-\frac{\alpha(p) \omega(p)}{p^{s}}\right)^{-1}\left(1-\frac{\beta(p) \omega(p)}{p^{s}}\right)^{-1}
$$

defined on the probability space $\left(\Omega, \mathcal{B}(\Omega), m_{H}\right)$.

Proof of Theorem 3. Using the Cauchy integral formula we obtain easily that the function $u: H\left(D_{V}\right) \rightarrow H\left(D_{V}\right)$ given by the formula $u(g(s))=g^{\prime}(s), g(s) \in H\left(D_{V}\right)$, is continuous. Therefore, [1, Theorem 5.1] and Lemma 4 imply, as $N \rightarrow \infty$, the weak convergence of the probability measure

$$
\mu_{N}\left(\varphi^{\prime}(s+i m h, F) \in A\right), \quad A \in \mathcal{B}\left(H\left(D_{V}\right)\right),
$$

to the distribution of the $H\left(D_{V}\right)$-valued random element $\varphi^{\prime}(s, \omega ; F)$. From this and Lemma 4, using a modification of the Cramér-Wald criterion (see, for example, [4], [6], [7], [9]) we obtain that the probability measure

$$
\mu_{N}\left(\left(\varphi^{\prime}(s+i m h ; F), \varphi(s+i m h ; F)\right) \in A\right), \quad A \in \mathcal{B}\left(H^{2}\left(D_{V}\right)\right),
$$

where $H^{2}\left(D_{V}\right)=H\left(D_{V}\right) \times H\left(D_{V}\right)$, converges weakly to the distribution of the $H^{2}\left(D_{V}\right)$-valued random element $\left(\varphi^{\prime}(s, \omega ; F), \varphi(s, \omega ; F)\right)$ as $N \rightarrow \infty$. The metric $d$ defined above satisfies $d\left(\frac{1}{g_{1}}, \frac{1}{d_{2}}\right)=d\left(g_{1}, g_{2}\right)$, and therefore the function $u: H^{2}\left(D_{V}\right) \rightarrow$ $M\left(D_{V}\right)$ defined by the formula $u\left(g_{1}, g_{2}\right)=\frac{g_{1}}{g_{2}}, g_{1}, g_{2} \in H\left(D_{V}\right)$, is continuous, and the theorem is a consequence of the weak convergence of the measure (1) as well as of [1, Theorem 5.1] again.

In order to use functional limit theorems for the proof of the universality property, it is necessary to know the support of the limit measure. In our limit theorems the limit measures coincide with the distributions of some random elements. Thus, we have to find the supports of those random elements.

THEOREM 4. The support of the random element $\frac{\varphi^{\prime}}{\varphi}(s, \omega ; F)$ is the whole of $H\left(D_{V}\right)$.

See [5, Lemma 7] for a proof of this theorem.

3. Discrete limit theorems. Case 2. In this section we suppose that the number $\exp \left\{\frac{2 \pi k}{h}\right\}$ is rational for some $k \neq 0$. Clearly, it suffices to consider only positive integers 
$k$. As it was noted in [3], there exists $k_{0} \in \mathbb{N}$ such that the numbers $k \in \mathbb{N}$ with the above property are the multiples of $k_{0}$. Let $A_{h}=\left\{k \in \mathbb{N} ; \exp \left\{\frac{2 \pi k}{h}\right\}\right.$ is rational $\}$, and let $k_{0}=\min _{k \in A_{h}} k$. If $k \in A_{h}$, then $k=a k_{0}+r$ with $0 \leq r<k_{0}$. Hence

$$
\exp \left\{\frac{2 \pi r}{h}\right\}=\exp \left\{\frac{2 \pi k}{h}\right\} \exp \left\{-\frac{2 \pi a k_{0}}{h}\right\}
$$

is rational. Therefore, by the definition of $k_{0}$ we have that $r=0$.

For $m \in \mathbb{N}$, we define

$$
\omega(m)=\sum_{p^{\alpha} \| m} \omega^{\alpha}(p)
$$

where $p^{\alpha} \| m$ means that $p^{\alpha} \mid m$ but $p^{\alpha+1} \nmid m$. Let

$$
\exp \left\{\frac{2 \pi k_{0}}{h}\right\}=\frac{m_{0}}{n_{0}}, \quad m_{0}, n_{0} \in \mathbb{N}, \quad\left(m_{0}, n_{0}\right)=1,
$$

and

$$
\Omega_{0}=\left\{\omega \in \Omega: \omega\left(m_{0}\right)=\omega\left(n_{0}\right)\right\} .
$$

Then $\Omega_{0}$ is a closed subgroup of $\Omega$, and therefore it is also a compact topological Abelian group. Hence we can define a probability space $\left(\Omega_{0}, \mathcal{B}\left(\Omega_{0}\right), m_{0 H}\right)$, where $m_{0 H}$ is the probability Haar measure on $\left(\Omega_{0}, \mathcal{B}\left(\Omega_{0}\right)\right)$. Denote the elements of $\Omega_{0}$ by $\omega_{0}$ and define

$$
\varphi\left(s, \omega_{0} ; F\right)=\prod_{p}\left(1-\frac{\alpha(p) \omega_{0}(p)}{p^{s}}\right)^{-1}\left(1-\frac{\beta(p) \omega_{0}(p)}{p^{s}}\right)^{-1} .
$$

By the same method as in the case of $\varphi(s, \omega ; F)$ it can be proved that $\varphi\left(s, \omega_{0} ; F\right)$ is an $H\left(D_{V}\right)$-valued random element defined on the probability space $\left(\Omega_{0}, \mathcal{B}\left(\Omega_{0}\right), m_{0 H}\right)$.

THEOREM 5. Suppose that $\exp \left\{\frac{2 \pi k}{h}\right\}$ is rational for some $k \neq 0$. Then the probability measure

$$
P_{N}(A) \stackrel{\text { def }}{=} \mu_{N}(\varphi(s+i m h ; F) \in A), \quad A \in \mathcal{B}\left(H\left(D_{V}\right)\right)
$$

converges weakly to the distribution of the random element $\varphi\left(s, \omega_{0} ; F\right)$ as $N \rightarrow \infty$.

Proof. The structure of the proof is similar to that in the case of $h$ when $\exp \left\{\frac{2 \pi k}{h}\right\}$ is irrational for all $k \in \mathbb{Z} \backslash\{0\}$. Therefore we will give a sketch of the proof only.

We start with a limit theorem on a finite-dimensional torus. Without loss of generality we can suppose that the prime numbers $p_{1}, \ldots, p_{l}$ occur in the factorization of the numbers $m_{0}$ and $n_{0}$, i.e., we have that

$$
\exp \left\{\frac{2 \pi k_{0}}{h}\right\}=\frac{m_{0}}{n_{0}}=p_{1}^{\alpha_{1}} \cdots p_{l}^{\alpha_{l}}
$$


with some $\alpha_{l} \in \mathbb{Z} \backslash\{0\}, j=1, \ldots, l$. Let $p_{1}, \ldots, p_{r}$ be all prime divisors of $n !, r>l$. Define a finite-dimensional torus

$$
\Omega_{r}=\prod_{j=1}^{r} \gamma_{p_{j}}
$$

where $\gamma_{p_{j}}=\gamma$ for $j=1, \ldots, r$, and let

$$
\Omega_{0 r}=\left\{\omega \in \Omega_{r}: \omega\left(m_{0}\right)=\omega\left(n_{0}\right)\right\},
$$

where for the extension of $\omega(p)$ to the set $\mathbb{N}, \omega \in \Omega_{r}$, formula (2) is used. Then in [3] it was proved that the probability measure

$$
Q_{h N}(A)=\mu_{N}\left(\left(p_{1}^{i m h}, \ldots, p_{r}^{i m h}\right) \in A\right), \quad A \in \mathcal{B}\left(\Omega_{r}\right),
$$

converges weakly to the Haar measure $m_{0 r}$ on $\left(\Omega_{0 r}, \mathcal{B}\left(\Omega_{0 r}\right)\right)$ as $N \rightarrow \infty$. Note that in this place the property of $h$ that $\exp \left\{\frac{2 \pi k}{h}\right\}$ is rational for some $k \neq 0$ is used. To prove the weak convergence of the measure $Q_{h N}$, we use the Fourier transform method. Denoting by $g_{N}\left(k_{1}, \ldots, k_{r}\right), k_{j} \in \mathbb{Z}, j=1, \ldots, r$, the Fourier transform of the measure $Q_{h N}$, we have that

$$
\begin{aligned}
g_{N}\left(k_{1}, \ldots, k_{r}\right) & =\frac{1}{N+1} \sum_{m=0}^{N} \prod_{j=1}^{r} p_{j}^{i m h k_{j}}=\frac{1}{N+1} \sum_{m=0}^{N} \exp \left(i m h \sum_{j=1}^{r} k_{j} \log p_{j}\right) \\
& = \begin{cases}1, & \text { if } k_{1}=k \alpha_{1}, \ldots, k \alpha_{l}, k_{l+1}=\ldots=k_{r}=0, \\
\frac{1}{N+1} & \frac{1-\exp \left\{i(N+1) h \sum_{j=1}^{r} k_{j} \log p_{j}\right\}}{1-\exp \left\{h i \sum_{j=1}^{r} k_{j} \log p_{j}\right\}} \quad \text { otherwise. }\end{cases}
\end{aligned}
$$

Note that in view of the linear independence over the field of rational numbers of logarithms of prime numbers and (3),

$$
1-\exp \left(h i \sum_{j=1}^{r} k_{j} \log p_{j}\right) \neq 0
$$

for $\left(k_{1}, \ldots, k_{l}, k_{l+1}=\cdots=k_{r}\right) \neq\left(k \alpha_{1}, \ldots, k \alpha_{l}, 0, \ldots, 0\right), k \in \mathbb{Z}$. Therefore,

$\lim _{N \rightarrow \infty} g_{N}\left(k_{1}, \ldots, k_{r}\right)= \begin{cases}1, & \text { if }\left(k_{1}, \ldots, k_{l}, k_{l+1}=\cdots=k_{r}\right) \neq\left(k \alpha_{1}, \ldots, k \alpha_{l}, 0, \ldots, 0\right), \\ 0, & \text { otherwise. }\end{cases}$

This implies the weak convergence of $Q_{h N}$ to $m_{0 r}$ as $N \rightarrow \infty$.

The proof thereafter contains the following sequence of statements: limit theorems in the space of analytic functions for Dirichlet polynomials and for absolutely convergent Dirichlet series, approximations in the mean by absolutely convergent series, limit theorems in the space of analytic functions for $\varphi(s ; F)$ and $\varphi\left(s, \omega_{0} ; F\right)$, and the identification of the limit measure. For Matsumoto zeta-functions, all these statements for the complex plane were proved in [3]. Here we have to generalize the mentioned statements for the space of analytic functions.

Let

$$
p_{n}(s)=\sum_{k=1}^{n} a_{k} k^{-s}, \quad a_{k} \in \mathbb{C}
$$


and

$$
p_{n}\left(s, \omega_{0}\right)=\sum_{k=1}^{n} a_{k} \omega_{0}(k) k^{-s}
$$

where $\omega_{0}$ is an element of $\Omega_{0}$, be two Dirichlet polynomials. Then, taking into account the weak convergence of the measure $Q_{h N}$ and [1, Theorem 5.1], similar to the proofs of [3, Theorems 4 and 5] we deduce that the probability measures

$$
\mu_{N}\left(p_{n}(s+i m h) \in A\right), \quad A \in \mathcal{B}\left(H\left(D_{V}\right)\right),
$$

and

$$
\mu_{N}\left(p_{n}\left(s+i m h, \omega_{0}\right) \in A\right), \quad A \in \mathcal{B}\left(H\left(D_{V}\right)\right),
$$

both converge weakly to the same probability measure on $\left(H\left(D_{V}\right), \mathcal{B}\left(H\left(D_{V}\right)\right)\right)$ as $N \rightarrow \infty$.

Now let, for $\sigma_{1}>1 / 2$ and $n \in \mathbb{N}$,

$$
\varphi_{n}(s ; F)=\sum_{m=1}^{\infty} \frac{c(m)}{m^{s}} \exp \left\{-\left(\frac{m}{n}\right)^{\sigma_{1}}\right\}
$$

and

$$
\varphi_{n}\left(s, \omega_{0} ; F\right)=\sum_{m=1}^{\infty} \frac{c(m) \omega_{0}(m)}{m^{s}} \exp \left\{-\left(\frac{m}{n}\right)^{\sigma_{1}}\right\} .
$$

The latter series both converge absolutely for $\sigma>\kappa / 2$. Then the analogue of [3, Theorem 6] says that there exists a probability measure $P_{n}$ on $\left(H\left(D_{V}\right), \mathcal{B}\left(H\left(D_{V}\right)\right)\right)$ such that both the measures

$$
\mu_{N}\left(\varphi_{n}(s+i m h ; F) \in A\right), \quad A \in \mathcal{B}\left(H\left(D_{V}\right)\right),
$$

and

$$
\mu_{N}\left(\varphi_{n}\left(s+i m h, \omega_{0} ; F\right) \in A\right), \quad A \in \mathcal{B}\left(H\left(D_{V}\right)\right),
$$

converge weakly to $P_{n}$ as $N \rightarrow \infty$. To prove this, instead of the Euclidean metric used in [3] we have to deal with a metric $\rho$ in $H\left(D_{V}\right)$ which induces its topology. There exists a sequence $\left\{K_{l}\right\}$ of compact subsets of $D_{V}$ such that

$$
D_{V}=\bigcup_{l=1}^{\infty} K_{l}
$$

$K_{l} \subset K_{l+1}$, and if $K$ is a compact subset of $D_{V}$, then $K \subseteq K_{l}$ for some $l$. Then the metric $\rho$ can be defined by

$$
\rho\left(g_{1}, g_{2}\right)=\sum_{l=1}^{\infty} 2^{-l} \frac{\rho_{l}\left(g_{1}, g_{2}\right)}{1+\rho_{l}\left(g_{1}, g_{2}\right)}, \quad g_{1}, g_{2} \in H\left(D_{V}\right),
$$


where

$$
\rho_{l}\left(g_{1}, g_{2}\right)=\sup _{s \in K_{l}}\left|g_{1}(s)-g_{2}(s)\right|
$$

Moreover, we use the weak convergence of probability measures (4) and (5) as well as [1, Theorem 4.2].

To pass from $\varphi_{n}(s ; F)$ to $\varphi(s ; F)$ an approximation of $\varphi(s ; F)$ by $\varphi_{n}(s ; F)$ in the mean is applied. Using the estimate ([11], [12])

$$
\int_{0}^{T}|\varphi(\sigma+i t ; F)|^{2} d t \ll T, \quad \sigma>\frac{\kappa}{2}
$$

we obtain that, for every compact subset $K \subset D_{V}$,

$$
\lim _{n \rightarrow \infty} \limsup _{N \rightarrow \infty} \frac{1}{N+1} \sum_{m=0}^{N} \sup _{s \in K}\left|\varphi(s+i m h ; F)-\varphi_{n}(s+i m h ; F)\right|=0 .
$$

The proof of a similar result for $\varphi\left(s, \omega_{0} ; F\right)$ is more complicated. Let $a_{h}=\left\{p^{-i h}\right.$ : $p$ is prime\}. Define a transformation $f_{h}$ of $\Omega_{0}$ taking $f_{h}\left(\omega_{0}\right)=a_{h} \omega_{0}, \omega_{0} \in \Omega_{0}$. Then $f_{h}$ is a measurable measure preserving transformation on $\left(\Omega_{0}, \mathcal{B}\left(\Omega_{0}\right), m_{0 H}\right)$. In [3] it was proved that the transformation $f_{h}$ is ergodic. This together with the classical Birkhoff theorem implies, for $\sigma>\kappa / 2$ and almost all $\omega_{0} \in \Omega$, the estimate

$$
\sum_{m=0}^{N}\left|\varphi\left(\sigma+i m h, \omega_{0} ; F\right)\right| \ll N .
$$

From this we find that, for every compact subset $K \subset D_{V}$,

$$
\lim _{n \rightarrow \infty} \limsup _{N \rightarrow \infty} \frac{1}{N+1} \sum_{m=0}^{N} \sup _{s \in K}\left|\varphi\left(s+i m h, \omega_{0} ; F\right)-\varphi_{n}\left(s+i m h, \omega_{0} ; F\right)\right|=0
$$

for almost all $\omega_{0} \in \Omega_{0}$.

Now the weak convergence of probability measures (6) and (7), the relations (8) and (9), and [1, Theorem 4.2] show that on $\left(H\left(D_{V}\right), \mathcal{B}\left(H\left(D_{V}\right)\right)\right)$ there exists a probability measure $P$ such that the probability measures $P_{N}$ and

$$
\mu_{N}\left(\varphi\left(s+i m h, \omega_{0} ; F\right) \in A\right), \quad A \in\left(H\left(D_{V}\right)\right),
$$

both converge weakly to $P$ as $N \rightarrow \infty$.

It remains to prove that the measure $P$ is the distribution of the random element $\varphi\left(s, \omega_{0} ; F\right)$. This is obtained by a standard way. Let $A \in \mathcal{B}\left(H\left(D_{V}\right)\right)$ be a continuity set of the measure $P$. Then the weak convergence of the measure (10) yields

$$
\lim _{N \rightarrow \infty} \mu_{N}\left(\varphi\left(s+i m h, \omega_{0} ; F\right) \in A\right)=P(A)
$$


for almost all $\omega_{0} \in \Omega_{0}$. For a fixed set $A$ define on the probability space $\left(\Omega_{0}\right.$, $\left.\mathcal{B}\left(\Omega_{0}\right), m_{0 H}\right)$ the random variable $\theta$ by

$$
\theta\left(\omega_{0}\right)= \begin{cases}1 & \text { if } \varphi\left(s, \omega_{0} ; F\right) \in A, \\ 0 & \text { if } \varphi\left(s, \omega_{0} ; F\right) \notin A .\end{cases}
$$

Denote by $\mathbb{E} \xi$ the expectation of the random element $\xi$. Then by the definition of $\theta$

$$
\mathbb{E} \theta=\int_{\Omega_{0}} \theta d m_{0 H}=m_{0 H}\left(\omega_{0} \in \Omega_{0}: \varphi\left(s, \omega_{0} ; F\right) \in A\right)=P_{\varphi}(A)
$$

is the distribution of $\varphi\left(s, \omega_{0} ; F\right)$. Since the transformation $f_{h}$ is ergodic, the Birkhoff theorem shows that

$$
\lim _{N \rightarrow \infty} \frac{1}{N+1} \sum_{m=0}^{N} \theta\left(f_{h}^{m}\left(\omega_{0}\right)\right)=\mathbb{E} \theta
$$

for almost all $\omega_{0} \in \Omega$. However, by the definitions of $f_{h}$ and $\theta$,

$$
\frac{1}{N+1} \sum_{m=0}^{N} \theta\left(f_{h}^{m}\left(\omega_{0}\right)\right)=\mu_{N}\left(\varphi\left(s+i m h, \omega_{0} ; F\right) .\right.
$$

Therefore, in view of (12) and (13),

$$
\lim _{N \rightarrow \infty} \mu_{N}\left(\varphi\left(s+i m h, \omega_{0} ; F\right)\right)=P_{\varphi}(A)
$$

for almost all $\omega_{0} \in \Omega_{0}$. Hence and from (11) we have that $P(A)=P_{\varphi}(A)$ for all continuity sets $A$ of the measure $P$. Therefore, $P(A)=P_{\varphi}(A)$ for all $A \in \mathcal{B}\left(H\left(D_{V}\right)\right)$. Theorem 5 is proved.

On the probability space $\left(\Omega_{0}, \mathcal{B}\left(\Omega_{0}\right), m_{0 H}\right)$ define an $H\left(D_{V}\right)$-valued random element $\varphi^{\prime}\left(s, \omega_{0} ; F\right)$ by the formula

$$
\begin{aligned}
\varphi^{\prime}\left(s, \omega_{0} ; F\right)= & \prod_{p}\left(1-\frac{\alpha(p) \omega_{0}(p)}{p^{s}}\right)^{-1}\left(1-\frac{\beta(p) \omega_{0}(p)}{p^{s}}\right)^{-1} \\
& \times\left(-\sum_{p} \frac{\alpha(p) \omega_{0}(p) \log p}{p^{s}}\left(1-\frac{\alpha(p) \omega_{0}(p)}{p^{s}}\right)^{-1}\right. \\
& \left.-\sum_{p} \frac{\beta(p) \omega_{0}(p) \log p}{p^{s}}\left(1-\frac{\beta(p) \omega_{0}(p)}{p^{s}}\right)^{-1}\right) .
\end{aligned}
$$

THEOREM 6. Suppose that $\exp \left\{\frac{2 \pi k}{h}\right\}$ is rational for some $k \neq 0$. Then the probability measure

$$
\mu_{N}\left(\varphi^{\prime}(s+i m h ; F) \in A\right), \quad A \in \mathcal{B}\left(H\left(D_{V}\right)\right),
$$

converges weakly to the distribution of the random element $\varphi^{\prime}\left(s, \omega_{0} ; F\right)$ as $N \rightarrow \infty$.

Proof. Since the function $u: H\left(D_{V}\right) \longrightarrow H\left(D_{V}\right)$ defined by $u(g(s))=g^{\prime}(s), g(s) \in$ $H\left(D_{V}\right)$, is continuous, the theorem is a consequence of Theorem 5 and Theorem 5.1 of $[1]$. 
Now on the probability space $\left(\Omega_{0}, \mathcal{B}\left(\Omega_{0}\right), m_{0 H}\right)$ define an $H\left(D_{V}\right)$-valued random element $\frac{\varphi^{\prime}}{\varphi}\left(s, \omega_{0} ; F\right)$ by the formula

$$
\begin{aligned}
\frac{\varphi^{\prime}}{\varphi}\left(s, \omega_{0} ; F\right)= & -\sum_{p} \frac{\alpha(p) \omega_{0}(p) \log p}{p^{s}}\left(1-\frac{\alpha(p) \omega_{0}(p)}{p^{s}}\right)^{-1} \\
& -\sum_{p} \frac{\beta(p) \omega_{0}(p) \log p}{p^{s}}\left(1-\frac{\beta(p) \omega_{0}(p)}{p^{s}}\right)^{-1} .
\end{aligned}
$$

THEOREM 7. Suppose that $\exp \left\{\frac{2 \pi k}{h}\right\}$ is rational for some $k \neq 0$. Then the probability measure

$$
\mu_{N}\left(\frac{\varphi^{\prime}}{\varphi}(s+i m h ; F) \in A\right), \quad A \in \mathcal{B}\left(M\left(D_{V}\right)\right),
$$

converges weakly to the distribution of the random element $\frac{\varphi^{\prime}}{\varphi}\left(s, \omega_{0} ; F\right)$ as $N \rightarrow \infty$.

Proof. The theorem is derived from Theorem 6 in the same way as the second part of the proof of Theorem 3 .

3. The supports of the random elements $\varphi^{\prime}\left(s, \omega_{0} ; \boldsymbol{F}\right)$ and $\frac{\varphi^{\prime}}{\varphi}\left(s, \omega_{0} ; \boldsymbol{F}\right)$. We start with a statement on the support of the random element $\varphi\left(s, \omega_{0} ; F\right)$. Let

$$
S_{V}=\left\{g \in H\left(D_{V}\right): g(s) \neq 0 \text { or } g(s) \equiv 0\right\} .
$$

LEMMA 8. The support of the random element $\varphi\left(s, \omega_{0} ; F\right)$ is the set $S_{V}$.

Proof. By the construction of the torus $\Omega,\{\omega(p)\}$ is a sequence of independent random variables defined on the probability space $\left(\Omega, \mathcal{B}(\Omega), m_{H}\right)$. Hence it follows easily that $\left\{\omega_{0}(p)\right\}$ is also a sequence of independent random variables defined on the probability space $\left(\Omega_{0}, \mathcal{B}\left(\Omega_{0}\right), m_{0 H}\right)$. Therefore, the proof of the lemma coincides with that of $[8$, Lemma 8$]$.

THEOREM 9. The support of the random element $\varphi^{\prime}\left(s, \omega_{0} ; F\right)$ is the whole of $H\left(D_{V}\right)$.

Proof. We repeat the arguments of the proof of Lemma 6. The function $u$ : $S_{V} \rightarrow H\left(D_{V}\right)$ defined by $u(g(s))=g^{\prime}(s), g(s) \in S_{V}$, is continuous. Hence the set $u^{-1} G$ is open for any open set $G \subset H\left(D_{V}\right)$. Using the metric $\rho$ defined in the proof of Theorem 5 and the Mergelyan theorem on uniform approximation of analytic functions by polynomials (see, for example, [14]), we ensure that the set $u^{-1} G \subset S_{V}$ is non-empty: there exists a polynomial $g(s) \in u^{-1} G$, and $g(s) \neq 0$ on $D_{V}$. Now we have by Lemma 8 that

$$
m_{0 H}\left(\omega_{0} \in \Omega_{0}:\left(\varphi\left(s, \omega_{0} ; F\right)\right)^{\prime} \in G\right)=m_{0 H}\left(\omega_{0} \in \Omega: \varphi\left(s, \omega_{0} ; F\right) \in u^{-1} G\right)>0 .
$$

Since the support of $\varphi^{\prime}\left(s, \omega_{0} ; F\right)$ consists of all $g \in H\left(D_{V}\right)$ such that for every neighborhood $G$ of $g$ the distribution $P_{\varphi^{\prime}}$ of $\varphi^{\prime}\left(s, \omega_{0} ; F\right)$ satisfies the inequality $P_{\varphi^{\prime}}(G)>0$, hence the theorem follows.

THEOREM 10. The support of the random element $\frac{\varphi^{\prime}}{\varphi}\left(s, \omega_{0} ; F\right)$ is the whole of $H\left(D_{V}\right)$. 
Proof. As it was noted above, $\left\{\omega_{0}(p)\right\}$ is a sequence of independent random variables on $\left(\Omega_{0}, \mathcal{B}\left(\Omega_{0}\right), m_{0 H}\right)$. Therefore, the proof of the theorem runs in the same way as that of [5, Lemma 7]: everywhere in place of $\omega(p)$ we write $\omega_{0}(p)$, only.

\section{Proof of Theorems 1 and 2.}

Proof of Theorem 1. We will consider only $\psi(s ; F)=\varphi^{\prime}(s ; F)$ in the second case. The remaining cases can be treated similarly.

First of all we observe that there exists a number $V>0$ such that $K \subset D_{V}$, and suppose that the function $f(s)$ is analytically continuable to the region $D_{V}$. Denote by $G$ the set of functions $g \in H\left(D_{V}\right)$ such that

$$
\sup _{s \in K}|g(s)-f(s)|<\varepsilon .
$$

By Theorem 9 the function $f(s)$ belongs to the support of the random element $\varphi^{\prime}\left(s, \omega_{0} ; F\right)$, moreover, the set $G$ is open. Therefore, by Theorem 6 , taking into account [1, Theorem 2.1, Statement 4] and the above-mentioned property of the support, we have that

$$
\liminf _{N \rightarrow \infty} \mu_{N}\left(\sup _{s \in K}\left|\varphi^{\prime}(s+i \tau ; F)-f(s)\right|<\varepsilon\right) \geq m_{0 H}\left(\omega_{0} \in \Omega_{0}: \varphi^{\prime}\left(s, \omega_{0} ; F\right) \in G\right)>0 .
$$

Now let $f(s)$ satisfy the hypothesis of Theorem 1. By the Mergelyan theorem, (see, for example, [15]), there exists a polynomial $p_{n}(s)$ such that

$$
\sup _{s \in K}\left|f(s)-p_{n}(s)\right|<\frac{\varepsilon}{2} .
$$

Since $p_{n}(s)$ is analytic in $D_{V}$, by the beginning of the proof it follows that

$$
\liminf _{N \rightarrow \infty} \mu_{N}\left(\sup _{s \in K}\left|\varphi^{\prime}(s+i \tau ; F)-p_{n}(s)\right|<\frac{\varepsilon}{2}\right)>0 .
$$

This and (14) yield the assertion of the theorem.

If $\psi(s ; F)=\frac{\varphi^{\prime}}{\varphi}(s ; F)$, then for the proof of Theorem 1 in the first and the second cases Theorems 3 and 4, and Theorems 7 and 10 are applied.

Proof of Theorem 2. As it was noted above, we have to consider only the second case. The investigation of the latter case is similar to that of the first one and we shall only sketch the arguments.

Define

$$
\widehat{\sigma}=\frac{\sigma_{1}+\sigma_{2}}{2}, \quad \sigma=\max \left\{\left|\sigma_{1}-\frac{2 \kappa+1}{4}\right|,\left|\sigma_{2}-\frac{2 \kappa+1}{4}\right|\right\}
$$

and we take $f(s)=s-\widehat{\sigma}$ and $0<\varepsilon<\frac{\sigma_{2}-\sigma_{1}}{20}$. Then in view of Theorem 1 there exists a constant $c=c\left(\sigma_{1}, \sigma_{2}\right)>0$ such that, for sufficiently large $N$,

$$
\mu_{N}\left(\max _{\left|s-\frac{2 \kappa+1}{4}\right| \leq \sigma_{0}}\left|\varphi^{\prime}(s+i m h ; F)-f(s)\right|<\varepsilon\right)>c .
$$


Since

$$
\left\{s \in \mathbb{C}:|s-\widehat{\sigma}|=\frac{\sigma_{2}-\sigma_{1}}{2}\right\} \subset\left\{s \in \mathbb{C}:\left|s-\frac{2 \kappa+1}{4}\right| \leq \sigma_{0}\right\},
$$

we have that, for $m$ satisfying (15),

$$
\max _{|s-\widehat{\sigma}|=\frac{\sigma_{2}-\sigma_{1}}{2}}\left|\varphi^{\prime}(s+i m h ; F)-(s-\widehat{\sigma})\right|<\frac{\sigma_{2}-\sigma_{1}}{20} .
$$

Therefore, the functions $s-\widehat{\sigma}$ and $\varphi^{\prime}(s+i m h)-(s-\widehat{\sigma})$ on the $\operatorname{disc}\{s \in \mathbb{C}:|s-\widehat{\sigma}| \leq$ $\left.\frac{\sigma_{2}-\sigma_{1}}{2}\right\}$ satisfy the hypotheses of Rouché's theorem. Since the function $s-\widehat{\sigma}$ on this disc has exactly one zero, by Rouché's theorem the function $\varphi^{\prime}(s+i m h ; F)$ on this disc also has one zero. By (15) the number of such $m, 0 \leq m \leq N$, for sufficiently large $N$, is greater than $c N$. This proves the theorem.

ACKNOWLEDGEMENT. The work was partially supported by Lithuanian Foundation of Studies and Science.

\section{REFERENCES}

1. P. Billingsley, Convergence of Probability Measures (John Wiley and Sons, New York, 1968).

2. P. Deligne, La conjecture de Weil I, Publ. I. H. E. S. 43 (1974), 273-307.

3. R. Kačinskaite and A. Laurinčikas, On the value distribution of the Matsumoto zetafunction, Acta Math. Hungar. 105 (2004), 339-359.

4. A. Laurinčikas, A joint limit theorem for zeta-functions attached to certain cusp forms, Publ. Math. Debrecen 59 (2001), 175-186.

5. A. Laurinčikas, On the derivatives of zeta-functions of certain cusp forms, Glasgow Math. J. 47 (2005), 87-96.

6. A. Laurinčikas and R. Garunkštis, The Lerch Zeta-Function (Kluwer Academic Publishers, Dordrecht, Boston, London, 2002).

7. A. Laurinčikas and K. Matsumoto, Joint value-distribution theorems on Lerch zetafunctions, Liet. Matem. Rink. 38 (1998), 312-326 (=Lith. Math. J. 38 (1998), 238-249).

8. A. Laurinčikas and $\mathrm{K}$. Matsumoto, The universality of zeta-functions attached to certain cusp forms, Acta Arith. 98 (2001), 345-359.

9. A. Laurinčikas and K. Matsumoto, The joint universality of zeta-functions attached to certain cusp forms, Fiz. Mat. Fak. Moksl. semin. Darb. 5 (2002), 58-75.

10. A. Laurinčikas, K. Matsumoto and J. Steuding, The discrete universality of $L$-functions of newforms, Math. Notes (submitted).

11. K. Matsumoto, A probabilistic study on the value-distribution of Dirichlet series attached to certain cusp forms, Nagoya Math. J. 116 (1989), 123-138.

12. K. Matsumoto, Recent developments in the mean square of the Riemann zeta and other zeta-functions, in Number Theory (ed. R. P. Bambah, V. C. Dumir and R. J. Hans-Gill), (Birkhäuser, 2000), 241-286.

13. A. Reich, Werteverteilung von Zetafunktionen, Arch. Math. (Basel) 34 (1980), 440-451.

14. S. M. Voronin, Theorem on the "universality" of the Riemann zeta-function, Izv. Akad. Nauk. SSSR, Ser. matem. 39 (1975), 475-486 (in Russian) = Math. USSR Izv. 9 (1975), 443-453.

15. J. L. Walsh, Interpolation and approximation by rational functions in the complex domain, Amer. Math. Soc. Coll. Pub., 1960. 